\title{
JEAN-BAPTISTE VENTURI (1746-1822)
}

Il y a deux cents ans, naissait à Bibiano, dans le duché de Reggio, en Italie, Jean-Baptiste Venturi. Professeur de métaphysique et de géométrie au séminare de cette ville à laage de 23 ans, il fut chargé, en 1773 , de la chaire de philosophie de Modène, et, bientot après, des fonctions d'ingénieur de ce petit Etat.

Une contestation très vive s'étant élevée en 1788 entre deux particuliers au sujet d'un partage d'eau pour l'irrigation, VENTusi fut entrainé à y prendre park. L'écrit qu’il fit paraître dans cetto afTaire « Riposta a quanto è stato scritto contro la relazione sulle irrigazioni del territorio di Castelnovo Gherardini », est plein de force et de raison et constitue sa première manifestation dans le domaine hydraulique.

Envoyé à Paris en 1796, à la suite du Comle de San-Romano, il y resta comme simple particulier pour se livrer entierement aux sciences, lut plusieurs mémoires à I'Institut et donna aux Annates de Chimie, au Journal des Mines et au Magasin Encyclopédique, quelques extraits d'ouvages scientifiques.

De relour dans sa patrie, il fut nommé membre du corps législatif de Milan, et, plus tard, professeur à l'Ecole du Génie à Modène. Le renversement du gouvernement républicain en 1799 lui conta la liberté qu'il ne recouvra qu'après la bataille de Marengo. La chaire de physique de l"Université de Pavie ei les décorations chevaleresques lui firent oublier sa disgràce. Il fut, pendant 12 ans, le chargé d'allaires du royaume d'ltalie à Berne, et mourut à Reggio en 1822.

Esprit éclairé, Vanteni est surtout comnu des hydrauliciens par son « Mémoire sur la transmission latérale du mouvement dans les fluides » publié en français à la fin du XVIII sicele.

La première planche de ce mémoire est consacrée par moitié à l'étude de ce que la postérité appela « l'eflet Venturi ». Cette étude n'est pas originale, l'équation de Bernouilli en est à l'origine et l'on retrouve le phénomène dans la figure 74 de l'Hydrodinamica, mais Vexturı étudia les conditions variées dans lesquelles il peut naître et indiqua ses plus importantes applications.

Le mémoire est axé sur l'idée centrale que tout jet fluide lancé à travers une masse fluide entrâne, par frottement, une portion plus ou moins importante de cette dernière. Après avoir donné des exemples de communication latérale de mouvement dans les fluides, l'auteur se demande si cette communication est produite par la visco. sité, e'est-à-dire par l'adhérence mutuelle des particules du fluide, ou bien par la dispersion du jet qui occasionne un mélange de ces particules.

Vexter s'engage ensuite dans l'examen de quelques applications du principe de l'entrainement des fluides par les fluides et l'étude de "l'effet Poleni » le mènera à l'effet qui porte son nom.

Le mémoire contient d'autres études d'un grand intérêt. Citons entre autres : l'évolution de la trompe catalane à la trompe Venturi et au distpositif étévatoire à ressaut, dont le champ d'applications modernes est extrêmement vaste, dans le domaine des partiteur's de débit pour l'irrigation, l'étude des tourbillons, en particulier le phénomène du Vortex ou tourbillon fixe à débit axial.

II ne nous appartient pas d'écrire, ici, une longue étude sur Jean-Baptiste Visnruk et son (x)uve, mais il convenait de marquer par quelques mots l'anniversaire de celui qui restera, selon le mot de M. Gariel dans l"article qu'il publia en 1935 ( ${ }^{2}$ ) un « jalon sur la route de l'hydraulique ».

(1) Revue Générale de l'Hydraulique. 